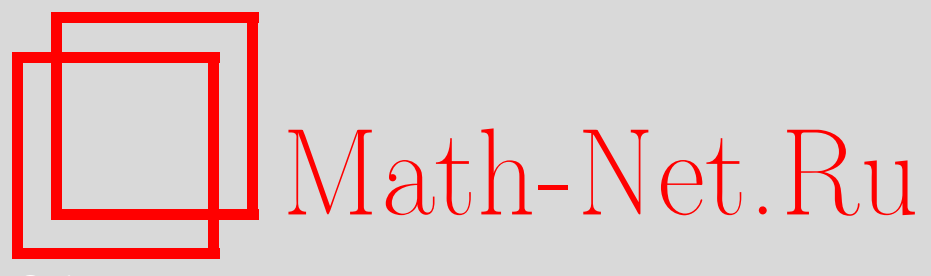

С. Ю. Доброхотов, Б. Тироцци, Локализованные решения одномерной нелинейной системы уравнений мелкой воды со скоростью $c=\sqrt{x}, У M H, 2010$, том 65, выпуск 1, 185-186

DOI: https://doi.org/10.4213/rm9342

Использование Общероссийского математического портала Math-Net.Ru подразумевает, что вы прочитали и согласны с пользовательским соглашением http://www . mathnet.ru/rus/agreement

Параметры загрузки:

IP : 54.162 .127 .20

26 апреля 2023 г., 18:30:46

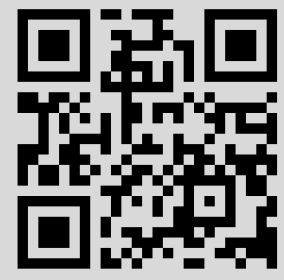




\title{
Локализованные решения одномерной нелинейной системы уравнений мелкой воды со скоростью $c=\sqrt{x}$
}

\author{
С. Ю. Доброхотов, Б. Тироцци
}

В безразмерных переменных нелинейная одномерная система уравнений мелкой воды над неровным дном $D(x)=c^{2}(x)$ для превышения $\eta(x, t)$ и скорости $v(x, t)$ имеет вид $\eta_{t}+\partial[v(\eta+D)] / \partial x=0, v_{t}+v v_{x}+\eta_{x}=0$. Введем параметр $0<\mu \ll 1$ и назовем функцию $f(y)$ локализованной в $\mu$-окрестности точки $a>0$, если $f(a)=1+O(\mu)$ и $f(y)=o(\mu)$ при $|y-a|>\mu^{1-\delta}, \delta>0$. В ситуации, когда $c^{2}(x)=x$, для этой системы рассмотрим задачу Коши $\left.\eta\right|_{t=0}=\eta^{0}(x, \mu),\left.v\right|_{t=0}=v^{0}(x, \mu)$ с начальными данными $\eta^{0}, v^{0}$, локализованными в окрестности точки $x=a$. Ее решение используется при описании набега длинных волн на берег [1], [2]. Прямым дифференцированием можно установить следующее замечательное свойство рассматриваемой системы, обнаруженное в работе [1] (хотя и представленное в другом виде, см. также [2]).

УтвеРЖДЕнИЕ 1. Пусть $(N(y, \tau), U(y, \tau))$ - решение линеаризованных уравнений мелкой води $N_{\tau}+\partial(y U) / \partial y=0, U_{\tau}+N_{y}=0$, определяющее гладкое решение $(\tau(t, x), y(t, x))$ системы $x=y-N(y, \tau)+(1 / 2) U^{2}(y, \tau), t=\tau+U(y, \tau)$. Тогда $(\eta, v)=$ $\left.\left(N-(1 / 2) U^{2}, U\right)\right|_{\tau=\tau(t, x), y=y(t, x)}$ - решение исходной нелинейной системы.

При изучении решений линейной системы уравнений на полуоси $y \geqslant 0$ с сингулярным коэффициентом $c^{2}=y$ требуем их ограниченности в точке $y=0$. (Это гарантирует принадлежность решений области определения оператора $\frac{\partial}{\partial y} y \frac{\partial}{\partial y}$.) В работах, касающихся рассматриваемых систем, по большей части изучаются их осциллирующие решения, локализованным решениям посвящены работы Р. Мазовой, Е. Пелиновского и их соавторов, см. статью [2] и библиографию в ней. Цель настоящего сообщения - построение простых точных решений линейной (а значит, и нелинейной) системы уравнений мелкой воды, а также интерпретация некоторых результатов [2].

УтверЖдение 2. Для любых комплексных чисел $A, b, \operatorname{Re} b \neq 0, u$ полинома $\mathscr{P}(k)$ непрерывные при $y \geqslant-(\operatorname{Re} b)^{2} / 4$ функции $\left(N^{0}, U^{0}\right)=\left(\operatorname{Re} \frac{A(\tau+i b)}{\left(y-(\tau+i b)^{2} / 4\right)^{3 / 2}}\right.$, $\left.2 \operatorname{Re} \frac{A}{\left(y-(\tau+i b)^{2} / 4\right)^{3 / 2}}\right) u(N, U)=\left(\mathscr{P}\left(\frac{\partial}{\partial \tau}\right) N^{0}, \mathscr{P}\left(\frac{\partial}{\partial \tau}\right) U^{0}\right)$ являются точньми решениями линейных уравнений мелкой водъ при $y \geqslant 0$. Если матрица Якоби перехода от $(\tau, y) \kappa(t, x)$ невырождена, то эти функции, в силу утверждения 1 , определяют точные параметрически заданные решения исходной нелинейной системы.

Доказательство утверждения 2 получается прямым дифференцированием, мы, однако, приведем и другие соображения, поясняющие как вывод, так и свойства приведенных решений. Выберем $b=\mu \beta / \sqrt{a}+2 i \sqrt{a}, A=\mu^{3 / 2}(1+i) /(2 \sqrt{a})$, тогда при $\tau=0$ построенные функции будут локализованы в $O(\mu)$-окрестности точки $y=a$. Рассмотрим более общую задачу Коши $\left.N\right|_{\tau=0}=V((y-a) / \mu),\left.U\right|_{\tau=0}=0$ для линейной системы. Здесь $V(z)$ - финитная функция $z$. Ввиду наличия параметра $\mu$, можно построить асимптотику решения при $\mu \rightarrow+0$ для этой системы (см. [2], [3]). Как и в известной формуле Даламбера, асимптотика решения распадается на две волны, одна из которых бежит направо, а вторая, $N_{\mathrm{L}}$, - налево. Амплитуда у $N_{\mathrm{L}}$ резко возрастает при приближении к точке $y=0$, которую поэтому разумно считать фокалъной точкой и аналогично [4] определить траекторию "пика" волны сразу на некомпактном "лагранжевом многообразии" $\Lambda=\left\{(p, x) \in \mathbb{R}_{p, y}^{2}: c(y) p^{2}=c^{2}(a)\right\}$, состоящем из двух кривых, заданных на фазовой плоскости $\mathbb{R}_{p, y}^{2}$. На $\Lambda$ движению

Работа выполнена при поддержке РФФИ (грант № 09-01-12063-офи-м) и GNFM (Италия). 
“пика" волны соответствует траектория $Y(t) \equiv(\sqrt{a}+\gamma t / 2)^{2}, P(t) \equiv-\sqrt{a} /(\sqrt{a}-\gamma t / 2)$ одномерной гамильтоновой системы с гамильтонианом $H=c(y)|p|, y(0)=a, p(0)=1$. Глобальная асимптотика решения задачи Коши определяется [5] формулой $N_{\mathrm{L}}(y, \tau)=$ $\frac{1}{2} \operatorname{Re}\left(\int_{0}^{\infty} K_{\Lambda}^{\mu / \rho} e^{i t c(a) \rho / \mu} \sqrt{\rho} \widetilde{V}(\rho) d \rho\right)$, где $K_{\Lambda}^{h}-$ канонический оператор Маслова на $\Lambda$ с параметром $h=\mu / \rho$, начальной точкой $x=a, p=-1$, а $\widetilde{V}(\rho)=\frac{1}{2 \pi} \int_{-\infty}^{\infty} e^{-i \rho z} V(z) d z$. Положим $t_{\mathrm{cr}}=2 \sqrt{a} / \gamma$, тогда реализация канонического оператора с дополнительным интегрированием по $\rho$ (обеспечивающим переход от осциллирующих функций к убывающим [5]) приводит к следующим формулам. При $\tau<\tau_{\mathrm{cr}}-\varepsilon$ (до достижения волны $\varepsilon$-окрестности точки $y=0) N_{\mathrm{L}}=\frac{1}{2} \sqrt{\frac{c(a)}{c(Y(\tau))}} V\left(\frac{c(a)}{\mu} \frac{y-Y(\tau)}{c(Y(\tau))}\right)+O(\mu)$, при $\left|\tau-\tau_{\text {cr }}\right|<\varepsilon$ (волна отражается от $y=0$ )

$\left.N_{\mathrm{L}}=\operatorname{Re}\left(\frac{e^{\frac{i \pi}{4}} \sqrt{a}}{\sqrt{\pi \mu}} \int_{0}^{\infty} \int_{-\infty}^{\infty} \mathbf{e}\left(\frac{1}{p}\right) \frac{\sqrt{\rho} \widetilde{V}^{0}(\rho)}{p} \exp \left\{\frac{i a \rho}{\mu}\left[\frac{1}{p}-2+\frac{\tau}{\sqrt{a}}\right]\right\} e^{\frac{i p y \rho}{\mu}}\right\} d p d \rho\right)+O(\sqrt{\mu})$

$(\mathbf{e}(q)$ - "срезающая" функция, равная 1 в некоторой окрестности точки $q=0)$, и при $\tau>\tau_{\mathrm{cr}}+\varepsilon$ (после отражения волны от $y=0$ )

$N_{\mathrm{L}}=\frac{1}{2} \sqrt{\frac{c(a)}{c(Y(\tau))}} W\left(\frac{c(a)}{\mu} \frac{y-Y(\tau)}{c(Y(\tau))}\right)+O(\mu)$, где $W(z)=-\sqrt{\frac{2}{\pi}} \operatorname{Re}\left(i \int_{0}^{\infty} e^{i \rho z} \widetilde{V}(\rho) d \rho\right)$

есть преобразование Гильберта от функции $V(\theta)$, вызванное скачком индекса Маслова после перехода точки $(Y(t), P(t))$ с нижнего листа $\Lambda$ на его верхний лист.

Формула (1) определяет асимптотику решения для всех $\tau$, однако вне окрестности точки $y=0$, разумеется, более удобно пользоваться приведенными явными формулами, которые можно получить из (1) методом стационарной фазы по переменной $p$. Функцию $\mathbf{e}(1 / p)$, обеспечивающую сходимость интегралов, иногда можно убрать, тогда (1) - точное решение. В частности, выбирая $\widetilde{V}(\rho)=A \sqrt{\rho} e^{-\beta \rho}$, получаем "базовое" решение из утверждения 2 , остальные получаются применением "оператора рождения" $\partial / \partial \tau$. Функции из утверждения 2 дают, по-видимому, уникальный пример, когда взаимодействие локализованной волны с фокальной точкой явно описывается в элементарных функциях; при $\mu \rightarrow 0$ при соответствующей нормировке падающая волна переходит в $\delta$-функцию Дирака, отраженная - в функцию Сохоцкого (имеет место “метаморфоза разрыва"). Отметим также, что соответствующая функция $V$ описывает профиль решения задачи Коши для двумерной линеаризованной системы уравнений мелкой воды с начальными данными $A\left(1+\left((y-\alpha) /\left(\mu b_{1}\right)\right)^{2}+\left(y_{2} /\left(\mu b_{2}\right)\right)^{2}\right)^{-3 / 2}$, локализованными в окрестности точки $\left(y=\alpha, y_{2}=0\right), a \gg \mu$ (см. [6]).

\section{Список литературы}

[1] G.F. Carrier, H.P. Greenspan, J. Fluid Mech., 4 (1958), 97-109. [2] E. N. Pelinovsky, R. Kh. Mazova, Natural Hazards, 6:3 (1992), 227-249. [3] S. Yu. Dobrokhotov, S. O. Sinitsyn, B. Tirozzi, Russ. J. Math. Phys., 14:1 (2007), 28-56. [4] T. Vukašinac, P. Zhevandrov, Russ. J. Math. Phys., 9:3 (2002), 371-381. [5] S. Yu. Dobrokhotov, B. Tirozzi, C. A. Vargas, Russ. J. Math. Phys, 16:2 (2009), 228-245. [6] S. Ya. Sekerzh-Zenkovich, B. I. Volkov, Russ. J. Math. Phys., 16:1 (2009), 121-129.

\section{C. Ю. Доброхотов (S. Yu. Dobrokhotov)}

Институт проблем механики РАН

E-mail: dobr@ipmnet.ru

\section{Б. Тироцци (В. Tirozzi)}

University of Rome "La Sapienza"

E-mail: b.tirozzi@libero.it
Представлено И. М. Кричевером Принято редколлегией 18.12.2009 